\title{
MULTIPLICITY OF SOLUTIONS OF THE DIRICHLET PROBLEM FOR AN EQUATION WITH THE $p$-LAPLACIAN IN A THREE-DIMENSIONAL SPHERICAL LAYER
}

\author{
S. B. KOLONITSKII
}

\begin{abstract}
The equation $-\Delta_{p} u=u^{q-1}$ with zero Dirichlet condition on the boundary is considered in a three-dimensional spherical layer. The existence of arbitrarily many distinct positive solutions in a sufficiently thin layer is proved.
\end{abstract}

\section{INTRODUCTION}

For $1<p<\infty$, we consider the boundary-value problem

$$
-\Delta_{p} u=u^{q-1} \text { in } \Omega_{R}, \quad u>0 \text { in } \Omega_{R}, \quad u=0 \text { on } \partial \Omega_{R},
$$

where $\Omega_{R}=B_{R} \backslash B_{R-1}$ is a a spherical layer in $\mathbb{R}^{n}, \Delta_{p} u=\operatorname{div}\left(|\nabla u|^{p-2} \nabla u\right)$ is the $p$-Laplacian, and $p<q<p^{*}$, where $p^{*}$ is the limit Sobolev embedding exponent, which is determined from the relation $\frac{1}{p^{*}}=\left(\frac{1}{p}-\frac{1}{n}\right)_{+}$.

In the paper [1, it was proved that, for $n \neq 3$, in a sufficiently thin layer, the boundaryvalue problem (11) admits many solutions. Namely, for any natural $K$, there exists an $R_{0}=R_{0}(p, q, K)$ such that for all $R>R_{0}$ we can find $K$ nonequivalent (i.e., those that cannot be obtained from one another by rotations) solutions of the boundary-value problem (11). Previously such results were obtained in the paper [2] for $p=2$. In the two-dimensional case, these results were derived in [3] for $p=2$ and in [4 for an arbitrary $p$ such that $1<p<\infty$. In those papers, the solutions of (1) were obtained as the minima of the functional

$$
J[u]=\int_{\Omega_{R}}|\nabla u|^{p} d x /\left(\int_{\Omega_{R}}|u|^{q} d x\right)^{\frac{p}{q}}
$$

on function spaces with a certain symmetry group.

For $n=3$ this method does not work; in [5] it was proved that this approach cannot give more than five distinct solutions at all. The existence of many solutions of the boundary-value problem (11) for $n=3$ and $p=2$ was established in the paper [6. The solutions of (11) were obtained as the minimum points of the functional (2) under special additional restrictions.

In the present paper, we establish the existence of many solutions of problem (11) for $n=3$ and arbitrary $p$. Henceforth, by a solution we mean a generalized solution in $\stackrel{\circ}{W}_{p}^{1}\left(\Omega_{R}\right)$.

Several auxiliary statements are given in $\S 1$; in $\S 2$ we study the boundary-value problem (1).

2010 Mathematics Subject Classification. Primary 35J92.

Key words and phrases. p-Laplacian, existence of many solutions.

Supported by RFBR (grant no. 08-01-00748) and by the grant NSh-227.2008.1 for support of leading scientific schools. 
We introduce some notation: $f=o_{R}(1)$ means that $\lim _{R \rightarrow \infty} f=0$. All functions with subscript $R$ have support in $\Omega_{R}$ and are assumed to be extended to $\mathbb{R}^{n} \backslash \Omega_{R}$ by zero. The components of a vector $x \in \mathbb{R}^{n}$ have superscripts in the notation, for example, $x^{(2)}$. For an arbitrary closed subgroup $\mathcal{G}$ of the group $\mathcal{O}(n)$ of rotations, we denote

$$
B_{\mathcal{G}}(x, \rho)=\{g y \mid y \in B(x, \rho), g \in \mathcal{G}\},
$$

where $B(x, \rho)$ is the open ball of radius $\rho$ with center $x$.

\section{§1. Auxiliary statements}

Throughout this section, $n \geq 2$ is an arbitrary natural number.

Lemma 1 (Principle of concentrated compactness, a generalization of Lemma 3.3 in [6]). Let $G(s)$ be a positive function. Consider a sequence $u_{R}$ of $\mathcal{G}$-invariant functions, and assume that $\int_{\Omega_{R}} G\left(u_{R}\right)$ is finite for any $R$. Then (after passing to a subsequence) one of the following three conditions is fulfilled:

1) (Concentration). For any $\varepsilon>0$, there exist a sequence $\left\{x_{R}\right\} \subset \mathbb{R}^{n}$ and a radius $\rho>0$ such that

$$
\int_{B_{\mathcal{G}}\left(x_{R}, \rho\right)} G\left(u_{R}\right) d x \geq(1-\varepsilon) \int_{\Omega_{R}} G\left(u_{R}\right) d x \text { for any } R .
$$

2) (Fuzzification). For all $\rho>0$, we have

$$
\lim _{R \rightarrow \infty} \sup _{y \in \mathbb{R}^{n}} \int_{B_{\mathcal{G}}(y, \rho)} G\left(u_{R}\right) d x=0 .
$$

3) (Separation). There exists $\lambda \in(0,1)$ such that for any $\varepsilon>0$, there exist $\rho>0$ and $R_{0}$ with the property that for any $R \geq R_{0}$ there are a sequence of points $\left\{x_{R}\right\} \subset \mathbb{R}^{n}$ and a sequence of numbers $\rho^{\prime}(R)$ tending to infinity and satisfying

$$
\begin{aligned}
& \left|\lambda \int_{\Omega_{R}} G\left(u_{R}\right) d x-\int_{B_{\mathcal{G}}\left(x_{R}, \rho\right)} G\left(u_{R}\right) d x\right| \\
& \quad+\left|(1-\lambda) \int_{\Omega_{R}} G\left(u_{R}\right) d x-\int_{\mathbb{R}^{n} \backslash B_{\mathcal{G}}\left(x_{R}, \rho^{\prime}(R)\right)} G\left(u_{R}\right) d x\right| \\
& \quad<\varepsilon \int_{\Omega_{R}} G\left(u_{R}\right) d x .
\end{aligned}
$$

Proof. Without loss of generality, we may assume that $\int_{\mathbb{R}^{n}} G\left(u_{R}\right)=1$. Consider the functions

$$
h(\rho, R)=\sup _{x \in \mathbb{R}^{n}} \int_{B_{\mathcal{G}}(x, \rho)} G\left(u_{R}\right) d x \quad \text { and } \quad i(\rho)=\limsup _{R \rightarrow \infty} h(\rho, R) ;
$$

$i(\rho)$ is a monotone nondecreasing and bounded function of $\rho$, and so we may consider $j=\lim _{\rho \rightarrow \infty} i(\rho)$.

If $j=0$, then $i(\rho)=0$ for any $\rho>0$, i.e., condition (4) is fulfilled.

If $j=1$, then for any $\varepsilon>0$, there is $\rho$ such that $i(\rho) \geq 1-\frac{\varepsilon}{3}$, i.e., there exists a subsequence $R_{m}$ with

$$
\sup _{x \in \mathbb{R}^{n}} \int_{B_{\mathcal{G}}(x, \rho)} G\left(u_{R_{m}}\right) d x \geq 1-\frac{2 \varepsilon}{3},
$$

and so we can find $x_{R}$ such that $\int_{B_{\mathcal{G}}(x, \rho)} G\left(u_{R_{m}}\right) d x \geq 1-\varepsilon$. In this case, condition (3) is satisfied (concentration).

Now, let $j=\lambda \in(0,1)$. We fix an arbitrary $\varepsilon>0$. Then there exists $\rho_{0}$ such that $i(\rho) \geq \lambda-\frac{\varepsilon}{6}$ if $\rho>\rho_{0}$. We fix such a $\rho>\rho_{0}$. Then $\lim \sup _{R \rightarrow \infty} h(\rho, R) \geq \lambda-\frac{\varepsilon}{6} \operatorname{implies}$ 
that there is a subsequence $R_{m}$ tending to infinity and such that $h\left(\rho, R_{m}\right) \geq \lambda-\frac{2 \varepsilon}{6}$, and thus there is a sequence of points $x_{m}$ with

$$
\int_{B_{\mathcal{G}}\left(x_{m}, \rho\right)} G\left(u_{R_{m}}\right) d x \geq \lambda-\frac{\varepsilon}{2} .
$$

On the other hand, $i(\rho) \leq \lambda$ for each $\rho$. Take a sequence $\rho_{m}$ tending to infinity. Then for any $m$ there exists $R_{0, m}$ such that for every $R>R_{0, m}$ we have $h\left(\rho_{m}, R\right) \leq \lambda+\frac{\varepsilon}{2}$, which means that

$$
\int_{B_{\mathcal{G}}\left(x, \rho_{m}\right)} G\left(u_{R_{m}}\right) d x \leq \lambda+\frac{\varepsilon}{2}
$$

for each $x \in \mathbb{R}^{n}$. Therefore,

whence

$$
\int_{B_{\mathcal{G}}\left(x_{m}, \rho\right)} G\left(u_{R_{m}}\right) d x \leq \int_{B_{\mathcal{G}}\left(x_{m}, \rho_{m}\right)} G\left(u_{R_{m}}\right) d x \leq \lambda+\frac{\varepsilon}{2},
$$

Moreover, from (7) we obtain

$$
\left|\lambda-\int_{B_{\mathcal{G}}\left(x_{m}, \rho\right)} G\left(u_{R_{m}}\right) d x\right| \leq \frac{\varepsilon}{2} .
$$

$$
\int_{\mathbb{R}^{n} \backslash B_{\mathcal{G}}\left(x, \rho_{m}\right)} G\left(u_{R_{m}}\right) d x \geq 1-\lambda-\frac{\varepsilon}{2} .
$$

On the other hand, (6) implies that

$$
\int_{\mathbb{R}^{n} \backslash B_{\mathcal{G}}\left(x, \rho_{m}\right)} G\left(u_{R_{m}}\right) d x \leq \int_{\mathbb{R}^{n} \backslash B_{\mathcal{G}}(x, \rho)} G\left(u_{R_{m}}\right) d x \leq 1-\lambda+\frac{\varepsilon}{2} .
$$

Thus,

$$
\left|1-\lambda-\int_{\mathbb{R}^{n} \backslash B_{\mathcal{G}}\left(x, \rho_{m}\right)} G\left(u_{R_{m}}\right) d x\right| \leq \frac{\varepsilon}{2} .
$$

Remark 1 (Concentration on an orbit). Let $x_{R}$ be a sequence of points in $\mathbb{R}^{n}$. Then similar arguments show that there exists a subsequence for which one of the following two statements is true:

1) for any $\rho>0$,

$$
\lim _{R \rightarrow \infty} \int_{B_{\mathcal{G}}\left(x_{R}, \rho\right)} G\left(u_{R}\right) d x=0 ;
$$

2) there exists $\lambda \in(0,1]$ such that for any $\varepsilon>0$ there are $\rho$ and $\rho^{\prime}(R)$ tending to infinity such that inequality (5) is fulfilled.

Remark 2. Two sequences of points $x_{R}$ and $y_{R}$ are said to be equivalent if there is $C>0$ such that the inequality $\min _{g \in \mathcal{G}}\left|x_{R}-g y_{R}\right|<C$ holds for every $R$. Obviously, an application of Remark 1 to the sequences $x_{R}$ and $y_{R}$ yields either (8) or (5) with one and the same $\lambda$. For this reason, in the sequel we do not distinguish between equivalent sequences.

Lemma 2. Let $u_{R}$ be a bounded sequence in $\stackrel{\circ}{W}_{p}^{1}\left(\mathbb{R}^{n}\right), p<q<p^{*}$, and

$$
\lim _{R \rightarrow \infty} \sup _{x \in \omega} \int_{B(x, \rho)}\left|u_{R}\right|^{q} d x=0
$$

for some $\rho>0$, where $\omega$ is an open set in $\mathbb{R}^{3}$ such that for all $x_{0} \in \omega$ we have

$$
\left|\omega \cap B\left(x_{0}, \rho\right)\right| \geq A \rho^{3}
$$

with $A$ independent of $\rho$ and $x_{0}$. Then $\int_{\omega}\left|u_{R}\right|^{q} d x \rightarrow 0$ as $R \rightarrow \infty$. 
Proof. By the Hölder inequality,

$$
\lim _{R \rightarrow \infty} \sup _{x \in \omega} \int_{B(x, \rho)}\left|u_{R}\right|^{p} d x=0 .
$$

We fix $l>0$ and denote

$$
A_{R, l}=\left\{x \in \mathbb{R}^{n} \mid u_{R}>l\right\} .
$$

By assumption, $\left|A_{R, l} \cap B(x, \rho) \cap \omega\right|$ tends to zero as $R \rightarrow \infty$ uniformly with respect to $x \in \omega$. By inequality (3.5) in [7, Chapter 2],

$$
\begin{aligned}
& \int_{A_{R, l} \cap B(x, \rho) \cap \omega}\left|u_{R}-l\right|^{p} d x \\
& \quad \leq \beta\left(\frac{\rho^{n}}{\left|B(x, \rho) \backslash\left(A_{R, l} \cap \omega\right)\right|}\right)^{p}\left|A_{R, l} \cap B(x, \rho) \cap \omega\right|^{\frac{p}{n}} \int_{A_{R, l} \cap B(x, \rho) \cap \omega}\left|\nabla u_{R}\right|^{p} d x,
\end{aligned}
$$

where $\beta$ depends only on $p, n$, and $q$. Hence, by the assumption $\left|A_{R, l} \cap B(x, \rho) \cap \omega\right| \rightarrow 0$, we obtain

$$
l^{p}\left|A_{R, 2 l} \cap B(x, \rho) \cap \omega\right| \leq 2 \beta\left|A_{R, l} \cap B(x, \rho) \cap \omega\right|^{\frac{p}{n}} \int_{A_{R, l} \cap B(x, \rho) \cap \omega}\left|\nabla u_{R}\right|^{p} d x
$$

for $R$ sufficiently large. We cover $\omega$ by balls $\left\{B\left(x_{i}, \rho\right)\right\}$ in such a way that any point $x \in \omega$ be contained in at most $2 n$ balls. Then, summing over all balls of this covering, we have

$$
l^{p}\left|A_{R, 2 l} \cap \omega\right| \leq 4 n \beta \sup _{i}\left|A_{R, l} \cap B\left(x_{i}, \rho\right) \cap \omega\right|^{\frac{p}{n}} \int_{A_{R, l} \cap \omega}\left|\nabla u_{R}\right|^{p} d x .
$$

Hence,

$$
l^{p}\left|A_{R, 2 l} \cap \omega\right| \rightarrow 0 \quad \text { as } \quad R \rightarrow \infty .
$$

Therefore,

$$
l^{p}\left|A_{R, l} \cap \omega\right| \rightarrow 0 \quad \text { as } \quad R \rightarrow \infty,
$$

and since $\int_{\Omega_{R}}\left|\nabla u_{R}\right|^{p} d x$ is bounded, we have

$$
\begin{aligned}
\int_{A_{R, l} \cap \omega}\left|u_{R}\right|^{p} d x & \leq 2^{p-1} \int_{A_{R, l} \cap \omega}\left(\left|u_{R}-l\right|^{p}+l^{p}\right) d x \\
& \leq C_{2} \sup _{i}\left|A_{R, l} \cap B\left(x_{i}, \rho\right) \cap \omega\right|^{\frac{p}{n}}+C_{1} l^{p}\left|A_{R, l} \cap \omega\right| .
\end{aligned}
$$

Thus,

$$
\int_{A_{R, l} \cap \omega}\left|u_{R}\right|^{p} d x \rightarrow 0 \quad \text { as } \quad R \rightarrow \infty .
$$

Using inequality (2.10) in [7, Chapter 2], we get

$$
\begin{aligned}
& \int_{\omega}\left|u_{R}\right|^{q} d x=\int_{\omega \backslash A_{R, l}}\left|u_{R}\right|^{q} d x+\int_{A_{R, l} \cap \omega}\left|u_{R}\right|^{q} d x \\
& \leq l^{q-p} \int_{\omega}\left|u_{R}\right|^{p} d x+C\left(\int_{A_{R, l} \cap \omega}\left|u_{R}\right|^{p} d x\right)^{\gamma_{1}}\left(\int_{\omega}\left|u_{R}\right|^{q_{1}} d x\right)^{1-\gamma_{1}} \\
& \leq l^{q-p} \int_{\omega}\left|u_{R}\right|^{p} d x+C\left(\int_{A_{R, l} \cap \omega}\left|u_{R}\right|^{p} d x\right)^{\gamma_{1}}\left(\int_{\omega}\left|u_{R}\right|^{p} d x\right)^{\gamma_{2}}\left(\int_{\omega}\left|\nabla u_{R}\right|^{p} d x\right)^{\gamma_{3}}
\end{aligned}
$$

(here $\gamma_{i}=\gamma_{i}(p, q)>0, i=1,2,3$, and $q<q_{1}<p^{*}$ ).

Taking into account the Friedrichs inequality and the boundedness of $\left\|\nabla u_{R}\right\|_{p}$, we see that $\int_{\omega} u_{R}^{p} d x \leq \int_{\omega}\left|\nabla u_{R}\right|^{p} d x \leq c$. The last summand tends to zero because the first of 
its factors tends to zero by inequality (10), and the second and third are bounded by the assumption of the lemma. Thus,

$$
\lim _{R \rightarrow \infty} \int_{\omega}\left|u_{R}\right|^{q} d x \leq c l^{q-p}
$$

where the constant $c$ does not depend on $l$. Therefore, choosing a sufficiently small $l$, for any $\varepsilon>0$ we have $\lim _{R \rightarrow \infty} \int_{\omega}\left|u_{R}\right|^{q} d x<\varepsilon$, i.e., $\lim _{R \rightarrow \infty} \int_{\omega}\left|u_{R}\right|^{q}=0$.

Remark 3. Let $u_{R}$ be a bounded sequence in $\stackrel{\circ}{W}_{p}^{1}\left(\mathbb{R}^{n}\right)$, and suppose that $\int_{\mathbb{R}^{n}}\left|u_{R}\right|^{q} d x$ is bounded away from zero with respect to $R$ for some $p<q<p^{*}$. Then

$$
\liminf _{R \rightarrow \infty} \sup _{x \in \mathbb{R}^{n}} \int_{B(x, \rho)}\left|u_{R}\right|^{q} d x>0 \text { for all } \rho>0 .
$$

\section{§2. Solution of Problem (1) FOR $n=3$}

Let $k$ be a fixed natural number. Consider the group $T_{k}$ generated by the orthogonal matrices

$$
\mathcal{R}_{k}=\left(\begin{array}{ccc}
\cos \frac{2 \pi}{k} & \sin \frac{2 \pi}{k} & 0 \\
-\sin \frac{2 \pi}{k} & \cos \frac{2 \pi}{k} & 0 \\
0 & 0 & 1
\end{array}\right) \quad \text { and } \quad \mathcal{T}=\left(\begin{array}{ccc}
1 & 0 & 0 \\
0 & 1 & 0 \\
0 & 0 & -1
\end{array}\right) ;
$$

$\mathcal{R}_{k}$ is rotation through the angle $\frac{2 \pi}{k}$, and $\mathcal{T}$ is reflection with respect to the equator. Also, we use the group $T_{\infty}$ generated by $\mathcal{T}$ and by all rotations about the axis $x^{(3)}=0$. Note that if a point $y$ does not lie on the equator and is not a pole, then $T_{\infty} y$ consists of two parallels symmetric about the equator, and $y$ lies on one of them. For brevity, we denote the set $B_{T_{k}}(x, \rho)$ by $B_{k}(x, \rho)$. Let $\mathcal{L}_{k}$ stand for the space of all $T_{k}$-invariant functions in $\stackrel{\circ}{W}_{p}^{1}\left(\Omega_{R}\right)$.

Consider the set

$$
\widetilde{\mathcal{L}_{k}}=\left\{\left.v \in \mathcal{L}_{k}\left|\int_{\Omega_{R}}\right| v\right|^{q} d x=1, \int_{\Omega_{R}} \chi_{\theta}|v|^{q} d x \leq \delta\right\},
$$

where

$$
\chi_{\theta}(r, \varphi, \theta)=\left\{\begin{array}{lll}
0 & \text { if } & \left|\theta-\frac{\pi}{2}\right|<\frac{\pi}{4}, \\
1 & \text { if } & \left|\theta-\frac{\pi}{2}\right| \geq \frac{\pi}{4},
\end{array}\right.
$$

and $\delta \in(0,1)$ is a fixed number. The boundary of the support of $\chi_{\theta}$ will be called the tropic.

We seek the minimum of the functional (2) on the set (12). Following [6], we prove that for sufficiently large $R$ the minimum is attained at a function $v_{R}$ satisfying the strict inequality $\int_{\Omega_{R}} \chi_{\theta}\left|v_{R}\right|^{q} d x<\delta$.

Lemma 3. The functional (2) attains its minimum on the set (12).

Proof. The set $\widetilde{\mathcal{L}_{k}}$ is weakly closed in $\stackrel{\circ}{W}_{p}^{1}\left(\Omega_{R}\right)$, because the embedding of $\stackrel{\circ}{W}_{p}^{1}\left(\Omega_{R}\right)$ in $L_{q}\left(\Omega_{R}\right)$ is compact. On the set $\widetilde{\mathcal{L}_{k}}$, the functional $J[u]$ coincides with the coercive convex functional $\|\nabla u\|_{p}^{p}$, so that it attains its minimum.

Remark 4. $\left|v_{R}\right|$ furnishes the minimum of the functional (2) in the same class of functions as $v_{R}$ does. Consequently, $\left|v_{R}\right| \geq 0$ is also a minimizer, and in the sequel we assume that $v_{R} \geq 0$. 
Lemma 4. Let $v_{R}$ be a sequence of $T_{k}$-invariant functions bounded in $\stackrel{\circ}{W}_{p}^{1}\left(\Omega_{R}\right)$, let $\left\|v_{R}\right\|_{q}=1$, and let

$$
\int_{\Omega_{R}} \chi_{\theta} v_{R}^{q} d x \leq \delta
$$

Then for any $\varepsilon>0$ there exists a sequence of $T_{k}$-invariant functions $W_{R}$ that has at most three concentration points, satisfies $1-\varepsilon \leq\left\|W_{R}\right\|_{q} \leq 1,\left\|\nabla W_{R}\right\|_{p} \leq\left\|\nabla v_{R}\right\|_{p}\left(1+o_{R}(1)\right)$, and inequality (14) holds for the functions $W_{R}$.

Proof. Since the sequence $v_{R}$ is bounded in $L_{q}\left(\mathbb{R}^{3}\right)$, one of the three statements, concentration, separation, and fuzzification with the function $G(s)=s^{q}$, is fulfilled for $v_{R}$.

Lemma 2 implies that fuzzification is impossible. Applying to $v_{R}$ symmetrization with respect to $\varphi$ on the interval $\left(-\frac{\pi}{k}, \frac{\pi}{k}\right)$, we do not change $\left\|v_{R}\right\|_{q}$ and do not increase $\left\|\nabla v_{R}\right\|_{p}$. Thus, we may assume that all concentration points lie on the plane $\left\{x^{(2)}=0\right\}$ in the quadrant $P=\left\{x^{(2)}=0, x^{(1)} \geq 0, x^{(3)} \geq 0\right\}$.

Consider the sequence $y_{R}=\left(R \sin \frac{\pi}{4}, 0, R \cos \frac{\pi}{4}\right)$. For this sequence we consider two cases described in Remark 1. In the first case, there are $\rho>0$ and a sequence $\rho^{\prime}(R)$ tending to infinity such that inequality (5) is fulfilled. We introduce a $T_{k}$-invariant cutoff function $\sigma$ :

$$
\begin{aligned}
& \sigma(x)= \begin{cases}1 & \text { for } x \in B_{k}\left(y_{R}, \rho\right), \\
1 & \text { for } x \notin B_{T_{\infty}}\left(y_{R}, \rho^{\prime}(R)\right), \\
0 & \text { for } x \in B_{T_{\infty}}\left(y_{R}, \rho^{\prime}(R)-\frac{\rho^{\prime}(R)-\rho}{4}\right) \backslash B_{k}\left(y_{R}, \rho^{\prime}(R)+\frac{\rho^{\prime}(R)-\rho}{4}\right),\end{cases} \\
& |\nabla \sigma| \leq \frac{8}{\rho^{\prime}(R)-\rho} .
\end{aligned}
$$

This cutoff function leaves intact a neighborhood of the concentration orbit $T_{k} y_{R}$ and a domain distant from the tropic. Without loss of generality, we may assume that $\rho^{\prime}(R) \leq \sqrt{R}$.

Then $\int_{\Omega_{R}}\left(\sigma v_{R}\right)^{q} d x \geq 1-\varepsilon$ and

$$
\begin{aligned}
& \int_{\Omega_{R}}\left|\nabla\left(\sigma v_{R}\right)\right|^{p} d x \\
& \quad \leq \int_{\Omega_{R}}\left|\nabla v_{R}\right|^{p} d x+C_{1}\left(\int_{\Omega_{R}}\left|\nabla v_{R}\right||\nabla \sigma|^{p-1} v_{R}^{p-1} \sigma d x+\int_{\Omega_{R}} v_{R}^{p}|\nabla \sigma|^{p} d x\right) .
\end{aligned}
$$

We estimate the last term:

$$
\begin{aligned}
\int_{\Omega_{R}} v_{R}^{p}|\nabla \sigma|^{p} d x & \leq C_{2}\left(\rho^{\prime}(R)-\rho\right)^{-p} \int_{\operatorname{supp} \nabla \sigma} v_{R}^{p} d x \\
& \leq C_{3}\left(\rho^{\prime}(R)-\rho\right)^{\left(\frac{3}{p}-\frac{3}{q}-1\right) p}\left(\int_{\Omega_{R}} v_{R}^{q} d x\right)^{\frac{p}{q}} \rightarrow 0
\end{aligned}
$$

as $R \rightarrow \infty$ because $\rho^{\prime}(R)-\rho \rightarrow \infty$ and $p<q<p^{*}$.

Then, by the Hölder inequality, the second term also tends to zero, and we have $\int_{\Omega_{R}} \nabla\left(\sigma v_{R}\right)^{p} d x=\int_{\Omega_{R}}\left|\nabla v_{R}\right|^{p} d x\left(1+o_{R}(1)\right)$.

In the second case, for any $\varepsilon>0$ there is a sequence $\rho^{\prime}(R)$ tending to infinity such that $\int_{B_{k}\left(y_{R}, \rho^{\prime}(R)\right)} v_{R}^{q} d x<\varepsilon$. In this case, we consider the following $T_{\infty}$-invariant cutoff function $\sigma$ :

$$
\sigma(x)=\left\{\begin{array}{ll}
1 & \text { for } x \notin B_{T_{\infty}}\left(y_{R}, \rho^{\prime}(R)\right), \\
0 & \text { for } x \in B_{T_{\infty}}\left(y_{R}, \frac{\rho^{\prime}(R)}{2}\right),
\end{array} \quad|\nabla \sigma| \leq \frac{4}{\rho^{\prime}(R)} .\right.
$$


As in the preceding case, we have

$$
\int_{\Omega_{R}}\left(\sigma v_{R}\right)^{q} d x \geq 1-\varepsilon \text { and } \int_{\Omega_{R}} \nabla\left(\sigma v_{R}\right)^{p} d x=\int_{\Omega_{R}}\left|\nabla v_{R}\right|^{p} d x\left(1+o_{R}(1)\right) .
$$

In both cases, the support of $\sigma$ contains the equatorial and two circumpolar connected components, and relations (16) hold true.

Consider two $T_{\infty}$-invariant cutoff functions: $\sigma_{1}$, which is equal to $\sigma$ on the connected components of $\operatorname{supp} \sigma$ that contain the poles and is equal to zero everywhere except for these components, and $\sigma_{2}$, which is equal to $\sigma$ on the connected component of $\operatorname{supp} \sigma$ that contains the equator and is equal to zero everywhere except for this component. Consider also the cutoff function $\sigma_{3}=\sigma-\sigma_{1}-\sigma_{2}$. The function $\sigma_{3}$ is $T_{k}$-invariant, and its support has $2 k$ connected components in the first case. In the second case, $\sigma_{3} \equiv 0$.

The cutoff functions $\sigma_{1}, \sigma_{2}$, and $\sigma_{3}$ single out the circumpolar, equatorial, and tropical concentration domains, respectively. We construct a function $W_{R}$ that has at most one concentration point in any of these domains.

Assume that the condition $\int_{\Omega_{R}}\left(\sigma_{1} v_{R}\right)^{q} d x \geq \lambda>0$ is satisfied for the sequence $\sigma_{1} v_{R}$. In each connected component of $\operatorname{supp} \sigma_{1} v_{R}$, we carry out spherical symmetrization with center at a pole and denote by $\widetilde{w}_{R}$ the function obtained. By symmetrization properties, we have $\left\|\widetilde{w}_{R}\right\|_{q}^{q}=\left\|\sigma_{1} v_{R}\right\|_{q}^{q}$ and $\left\|\nabla \widetilde{w}_{R}\right\|_{p} \leq\left\|\nabla\left(\sigma_{1} v_{R}\right)\right\|_{p}$. We apply Lemma 11 to the sequence $\widetilde{w}_{R}$. Lemma 2 shows that degeneration is impossible. Moreover, the sequence $\widetilde{w}_{R}$ has no concentration points other than the pole $N_{R}=(0,0, R)$. Applying Lemma 2 to the set $\mathbb{R}^{3} \backslash B_{k}\left(N_{R}, 2 \rho\right)$, we conclude that $\int_{\mathbb{R}^{3} \backslash B_{k}\left(N_{R}, 2 \rho\right)} \widetilde{w}_{R}^{q} d x \rightarrow 0$ as $R \rightarrow \infty$. Thus, there exists a $\rho_{1}>0$ such that

$$
\left|\int_{\Omega_{R}} \widetilde{w}_{R}^{q} d x-\int_{B_{k}\left(N_{R}, \rho_{1}\right)} \widetilde{w}_{R}^{q} d x\right|<\varepsilon \int_{\Omega_{R}} \widetilde{w}_{R}^{q} d x .
$$

Now, consider a $T_{\infty}$-invariant cutoff function $\tau_{1}$ such that

$$
\tau_{1}(x)=\left\{\begin{array}{ll}
1 & \text { for } x \in B_{k}\left(N_{R}, \rho_{1}+\sqrt{R}\right), \\
0 & \text { for } x \notin B_{k}\left(N_{R}, \rho_{1}+2 \sqrt{R}\right),
\end{array} \quad\left|\nabla \tau_{1}\right| \leq \frac{4}{\sqrt{R}} .\right.
$$

As above, we obtain

$$
\left\|\tau_{1} \widetilde{w}_{R}\right\|_{q}^{q} \geq(1-\varepsilon)\left\|\widetilde{w}_{R}\right\|_{q}^{q} \quad \text { and } \quad\left\|\nabla\left(\tau_{1} \widetilde{w}_{R}\right)\right\|_{p}^{p} \leq\left\|\nabla \widetilde{w}_{R}\right\|_{p}^{p}\left(1+o_{R}(1)\right) .
$$

Now we set $w_{R}=\tau_{1} \widetilde{w}_{R}$. If $\int_{\Omega_{R}}\left(\sigma_{1} v_{R}\right)^{q} d x \rightarrow 0$ as $R \rightarrow \infty$, then, to unify the notation, we set $w_{R} \equiv 0$. We also note that $\int_{\Omega_{R}} \chi_{\theta} w_{R}^{q} d x \leq \delta$.

Now we consider $\sigma_{2} v_{R}$. As before, there is no loss of generality in assuming that the concentration points lie only on $P$. Consider the set

$$
Q=\left\{g\left(r \cos \theta \cos \frac{\pi}{k}, r \cos \theta \sin \frac{\pi}{k}, r \sin \theta\right) \mid g \in T_{k}, r \in[R-1, R], \frac{\pi}{4} \leq \theta \leq \frac{3 \pi}{4}\right\} .
$$

In any finite neighborhood of $B(Q, \rho)$, the integral $\int_{B(Q, \rho)}\left(\sigma_{2} v_{R}\right)^{q} d x$ tends to zero, so that we can choose a sequence $D_{R}$ tending to infinity and such that $\int_{B\left(Q, D_{R}\right)}\left(\sigma_{2} v_{R}\right)^{q} d x<$ $\frac{\varepsilon}{2}$. Consider a cutoff function $\tau_{2}$ with the properties

$$
\widetilde{\tau}_{2}=\left\{\begin{array}{ll}
1 & \text { for } x \notin B\left(Q, D_{R}\right), \\
0 & \text { for } x \in B\left(Q, \frac{D_{R}}{2}\right),
\end{array} \quad\left|\nabla \widetilde{\tau}_{2}\right| \leq \frac{4}{D_{R}},\right.
$$

and the cutoff function $\tau_{2}=\frac{1}{2} \prod_{g \in T_{k}} \widetilde{\tau}_{2}(g x)$. Arguing as before, we get

$$
\left\|\tau_{2} \sigma_{2} v_{R}\right\|_{q}^{q} \geq(1-\varepsilon)\left\|\sigma_{2} v_{R}\right\|_{q}^{q} \quad \text { and } \quad\left\|\nabla\left(\tau_{2} \sigma_{2} v_{R}\right)\right\|_{p}^{p}=\left\|\nabla\left(\sigma_{2} v_{R}\right)\right\|_{p}^{p}\left(1+o_{R}(1)\right) \text {. }
$$


Introducing the cutoff function $\tau_{3}$ that is equal to $\tau_{2}$ on the connected component of $\operatorname{supp} \tau_{2}$ intersecting $P$ and is equal to zero at all remaining points, we apply spherical symmetrization to $\tau_{3} \sigma_{2} v_{R}$ and denote by $\widetilde{w}_{R}^{\prime}$ the function obtained. We have

$$
\int_{\Omega_{R}}\left(\widetilde{w}_{R}^{\prime}\right)^{q} d x=\frac{1}{k} \int_{\Omega_{R}}\left(\tau_{2} \sigma_{2} v_{R}\right)^{q} d x \quad \text { and } \quad \int_{\Omega_{R}}\left|\nabla \widetilde{w}_{R}^{\prime}\right|^{p} d x \leq \frac{1}{k} \int_{\Omega_{R}}\left|\nabla\left(\tau_{2} \sigma_{2} v_{R}\right)\right|^{p} d x .
$$

We apply to $\widetilde{w}_{R}^{\prime}$ the lemma on concentration (Lemma 1). As before, fuzzification and dichotomy are impossible; thus, condition (3) is satisfied. Consider a cutoff function $\tau_{4}$ such that

$$
\tau_{4}= \begin{cases}1 & \text { for } \quad x \in B((R, 0,0), \rho), \\ 0 & \text { for } x \notin B\left((R, 0,0), \rho+\frac{\rho^{\prime}(R)-\rho}{2}\right),\end{cases}
$$

and the $T_{k}$-invariant cutoff function $\tau_{5}=\frac{1}{2} \sum_{g \in T_{k}} \tau_{4}(g x)$. As before, we may assume that $\rho+\frac{\rho^{\prime}(R)-\rho}{2} \leq \sqrt{R}$, and we have

$$
\left\|\tau_{4} \widetilde{w}_{R}^{\prime}\right\|_{q}^{q} \geq(1-\varepsilon)\left\|\widetilde{w}_{R}^{\prime}\right\|_{q}^{q} \text { and } \quad\left\|\nabla\left(\tau_{4} \widetilde{w}_{R}^{\prime}\right)\right\|_{p}^{p}=\left\|\nabla \widetilde{w}_{R}^{\prime}\right\|_{p}^{p}\left(1+o_{R}(1)\right) .
$$

We set $w_{R}^{\prime}=\tau_{4} \widetilde{w}_{R}^{\prime}$ and note that the supports of $w_{R}^{\prime}(x)$ and $w_{R}^{\prime}\left(\mathcal{R}_{k}^{j} x\right)$ do not intersect if $j$ is not a multiple of $k$.

In the case where $\sigma_{3} \neq 0$, we consider an additional cutoff function that distinguishes one connected component of $\operatorname{supp} \sigma_{3}$ :

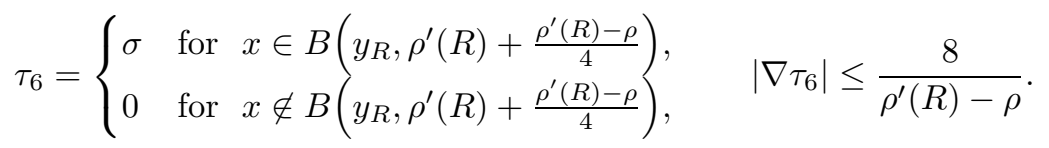

Then $\sigma_{3}=\sum_{g \in T_{k}} \tau_{6}(g x)$. The rotation $\mathcal{S}$ about the axis $x^{(2)}$ through the angle $\frac{\pi}{8}$ takes the point $N_{R}$ to the point $\left(R \cos \frac{3 \pi}{8}, 0, R \sin \frac{3 \pi}{8}\right)$. To $\tau_{6} v_{R}(\mathcal{S} x)$ we apply spherical symmetrization with center at the point $\mathcal{S} y_{R}$, and we denote by $w_{R}^{\prime \prime}(x)$ the function obtained. If $\sigma_{3}=0$, we set $w_{R}^{\prime \prime} \equiv 0$ to unify the notation.

The graph of $w_{R}$ represents two caps with centers at the poles, the graph of $w_{R}^{\prime}$ is a cap with center on the equator, and the graph of $w_{R}^{\prime \prime}$ is a cap with center between the equator and the tropic.

Now we consider the function

$$
W_{R}(x)=w_{R}(x)+\frac{1}{2} \sum_{g \in T_{k}} w_{R}^{\prime}(g x)+\sum_{g \in T_{k}} w_{R}^{\prime \prime}(g x) .
$$

The properties of the functions $w_{R}, w_{R}^{\prime}$, and $w_{R}^{\prime \prime}$ show that $\left\|W_{R}\right\|_{q}^{q} \geq 1-4 \varepsilon,\left\|\nabla W_{R}\right\|_{p} \leq$ $\left\|v_{R}\right\|_{p}\left(1+o_{R}(1)\right)$, and $\int_{\Omega_{R}} \chi_{\theta} W_{R}^{q} d x \leq \delta$.

Lemma 5. Let $\delta=\frac{1}{k}$, and let $v_{R}$ minimize the functional $J$ on $\widetilde{\mathcal{L}_{k}}$. Then for sufficiently large $R$ we have

$$
\int_{\Omega_{R}} \chi_{\theta}\left|v_{R}\right|^{q} d x<\delta
$$

Proof. Assume the contrary: let the condition $\int_{\Omega_{R}} \chi_{\theta} v_{R}^{q} d x=\delta$ be valid for the sequence $v_{R}$. Suppose $W_{R}$ is constructed in the same way as in Lemma 4. 
We put

$$
\begin{aligned}
a_{R} & =\frac{1}{2} \int_{\Omega_{R}}\left|\nabla w_{R}\right|^{p} d x ; & \alpha_{R} & =\frac{1}{2} \int_{\Omega_{R}} w_{R}^{q} d x, \\
b_{R} & =\int_{\Omega_{R}}\left|\nabla w_{R}^{\prime}\right|^{p} d x ; & \beta_{R} & =\int_{\Omega_{R}} w_{R}^{\prime q} d x, \\
c_{R} & =\int_{\Omega_{R}}\left|\nabla w_{R}^{\prime \prime}\right|^{p} d x ; & \gamma_{R} & =\int_{\Omega_{R}} w_{R}^{\prime \prime q} d x,
\end{aligned}
$$

where $w_{R}, w_{R}^{\prime}$, and $w_{R}^{\prime \prime}$ are as in Lemma 4. By construction, we have

$$
1-\varepsilon \leq 2 \alpha_{R}+k \beta_{R}+2 k \gamma_{R} \leq 1, \quad 2 \alpha_{R} \leq \delta, \quad 2 \alpha_{R}+2 k \gamma_{R} \geq \delta-\varepsilon .
$$

Now we show that we can reduce the value of the functional. For this, among the functions $\chi_{\left\{x_{(3)}>0\right\}} w_{R}, w_{R}^{\prime}$, and $w_{R}^{\prime \prime}$ we choose a function at which the functional $J$ has the smallest value. Namely, consider the sequences $\alpha_{R}, \beta_{R}$, and $\gamma_{R}$. They cannot tend to zero simultaneously; therefore, after passing to subsequences, we may assume that one of them is bounded away from zero. Among the ratios $a_{R} / \alpha_{R}^{\frac{p}{q}}, b_{R} / \beta_{R}^{\frac{p}{q}}$, and $c_{R} / \gamma_{R}^{\frac{p}{q}}$, we choose those with denominators bounded away from zero, and among them we take the minimum one.

If this is $\alpha_{R}$, then we set $m_{R}=a_{R}, \mu_{R}=\alpha_{R}$, and $\widetilde{u}_{R}=\chi_{\left\{x_{(3)}>0\right\}} w_{R}\left(\mathcal{S}^{4} x\right)$. If this is $\beta_{R}$, then we set $m_{R}=b_{R}, \mu_{R}=\beta_{R}$, and $\widetilde{u}_{R}=w_{R}^{\prime}(x)$. If this is $\gamma_{R}$, then we put $m_{R}=c_{R}, \mu_{R}=\gamma_{R}$, and $\widetilde{u}_{R}=w_{R}^{\prime \prime}(\mathcal{S} x)$. Next, we consider the function

$$
\begin{aligned}
u_{R}(x)= & \left(\frac{\alpha_{R}}{\mu_{R}}\right)^{\frac{1}{q}}\left(\widetilde{u}_{R}\left(\mathcal{S}_{1}^{-4} x\right)+\widetilde{u}_{R}\left(\mathcal{S}_{1}^{4} x\right)\right) \\
& +\frac{1}{2}\left(\frac{\beta_{R}}{\mu_{R}}\right)^{\frac{1}{q}} \sum_{g \in T_{k}} \widetilde{u}_{R}(g x)+\left(\frac{\gamma_{R}}{\mu_{R}}\right)^{\frac{1}{q}} \sum_{g \in T_{k}} \widetilde{u}_{R}\left(g \mathcal{S}_{1}^{-1} x\right) .
\end{aligned}
$$

Thus, we have replaced all caps by caps of the best shape that have the same mass.

We have

$$
\begin{aligned}
\int_{\Omega_{R}} u_{R}^{q} d x & =2 \alpha_{R}+k \beta_{R}+2 k \gamma_{R} \leq 1 \\
\int_{\Omega_{R}}\left|\nabla u_{R}\right|^{p} d x & =\left(2 \alpha_{R}^{\frac{p}{q}}+k \beta_{R}^{\frac{p}{q}}+2 k \gamma_{R}^{\frac{p}{q}}\right) \frac{m_{R}}{\mu_{R}^{\frac{p}{q}}} \leq \int_{\Omega_{R}}\left|\nabla W_{R}\right|^{p} d x,
\end{aligned}
$$

and

$$
\left(2 \alpha_{R}^{\frac{p}{q}}+k \beta_{R}^{\frac{p}{q}}+2 k \gamma_{R}^{\frac{p}{q}}\right) \frac{m_{R}}{\mu_{R}^{\frac{p}{q}}} \leq J\left[u_{R}\right] \leq J\left[W_{R}\right] .
$$

We retain only the caps on the equator, i.e., we consider the function

$$
U_{R}(x)=\frac{1}{2} \sum_{g \in T_{k}} \widetilde{u}_{R}(g x) .
$$

We have

$$
\int_{\Omega_{R}} U_{R}^{q} d x=k \mu_{R}, \quad \int_{\Omega_{R}}\left|\nabla U_{R}\right|^{p} d x=k m_{R}
$$

and $J\left[U_{R}\right]=k^{1-\frac{p}{q}} m_{R} / \mu_{R}^{\frac{p}{q}}$.

Under conditions (18), the minimum value of the function $2 \alpha_{R}^{\frac{p}{q}}+k \beta_{R}^{\frac{p}{q}}+2 k \gamma_{R}^{\frac{p}{q}}$ is equal to $2^{1-\frac{p}{q}} \delta^{\frac{p}{q}}+k^{1-\frac{p}{q}}(1-\varepsilon-\delta)^{\frac{p}{q}}$. Therefore,

$$
J\left[U_{R}\right] \leq \frac{1}{\left(\frac{2}{k}\right)^{1-\frac{p}{q}} \delta^{\frac{p}{q}}+(1-\varepsilon-\delta)^{\frac{p}{q}}} J\left[u_{R}\right] .
$$


Since $\delta=\frac{1}{k}$, we have $\left(\frac{2}{k}\right)^{1-\frac{p}{q}} \delta^{\frac{p}{q}}+(1-\varepsilon-\delta)^{\frac{p}{q}}=\frac{2^{1-\frac{p}{q}}}{k}+\left(1-\frac{1}{k}-\varepsilon\right)^{1-\frac{p}{q}} \geq 1+\frac{2^{1-\frac{p}{q}}-1}{k}-\varepsilon \geq$ $1+\frac{2^{1-\frac{p}{q}}-1}{2 k}$ for sufficiently small $\varepsilon$. Then

$$
\begin{aligned}
J\left[U_{R}\right] & \leq \frac{1}{1+\frac{2^{1-\frac{p}{q}}-1}{2 k}} J\left[u_{R}\right] \\
& \leq \frac{1}{1+\frac{2^{1-\frac{p}{q}}-1}{2 k}} J\left[W_{R}\right] \leq \frac{1}{(1-\varepsilon)^{\frac{p}{q}}\left(1+\frac{2^{1-\frac{p}{q}}-1}{2 k}\right)} J\left[v_{R}\right]\left(1+o_{R}(1)\right) .
\end{aligned}
$$

Since $\int_{\Omega_{R}} \chi_{\theta} U_{R}^{q} d x=0$, for sufficiently small $\varepsilon$ we obtain a contradiction with the minimality of $J\left[v_{R}\right]$.

Remark 5. As a by-product, we have proved that the sequence $v_{R}$ may have only one concentration orbit lying on the equator.

Theorem 1. Let $\delta=\frac{1}{k}$. If $R$ is sufficiently large, then the function $v_{R}$ constructed in Lemma 3 , upon multiplication by an appropriate constant, is a positive generalized solution of the boundary-vale problem (1).

Proof. By Lemma [5] the restriction $\int_{\Omega_{R}} \chi_{\theta} v_{R}^{q} d x \leq \frac{1}{k}$ is inactive for large $R$. Therefore, $v_{R}$ is a local minimum on the set $\left\{\left.u \in \stackrel{\circ}{W}_{p}^{1}\left(\Omega_{R}\right)\left|\int_{\Omega_{R}}\right| u\right|^{q} d x=1\right\}$. By the EulerLagrange theorem, for some $\lambda \in \mathbb{R}$ we have

$$
\int_{\Omega_{R}}\left(\left(\left|\nabla v_{R}\right|^{p-2} \nabla v_{R}, \nabla h\right)-\lambda\left|v_{R}\right|^{q-2} v_{R} h\right) d x=0
$$

for all $h \in \mathcal{L}_{k}$.

By the symmetric criticality principle (see [8]), $v_{R}$ is a solution of the Dirichlet problem

$$
-\Delta_{p} u=\lambda u^{q-1} \text { in } \Omega_{R} ; \quad u=0 \text { on } \partial \Omega_{R} .
$$

It remains to note that $v_{R} \geq 0$ is super-p-harmonic, and, by the Harnack inequality (see [9]), it is positive in $\Omega_{R}$. Since the two sides of equation (20) have different degrees of homogeneity, the function $u=c v_{R}$ with an appropriate constant $c$ is a solution of (11).

Theorem 2. For any natural $N$, there exists $R_{0}=R_{0}(p, q, N)$ such that for any $R>R_{0}$, the problem (11) has at least $N$ nonequivalent (i.e., such that they cannot be taken to one another by rotations) positive solutions.

Proof. The boundary-value problem (1) has a $T_{k}$-invariant solution for any $1 \leq k \leq N$. As was proved in Lemma 5, for a sequence of such solutions condition (3) is satisfied, and the distance from $x_{R}$ to the equator is bounded. Without loss of generality, we may assume that $x_{R}=(R, 0,0)$. Moreover, for any $\varepsilon>0$ there is a $\rho>0$ such that for all $R>R_{0}(p, q, k)$ we have $\left|\int_{B\left(x_{R}, \rho\right)} v_{R}^{q} d x-\frac{1}{k}\right|<\varepsilon$. Therefore, for different $k$ the $v_{R}$ are distinct provided that $R$ is sufficiently large.

The author is grateful to Professor A. I. Nazarov for statement of the problem and permanent encouragement.

\section{REFERENCES}

[1] A. I. Nazarov, On solution to the Dirichlet problem for an equation with $p$-Laplacian in a spherical layer, Trudy S.-Peterburg. Mat. Obshch. 10 (2004), 33-62; English transl., Amer. Math. Soc. Transl. Ser. 2, vol. 214, Amer. Math. Soc., Providence, RI, 2005, pp. 29-57. MR2181511 (2006i:35101)

[2] Y. Y. Li, Existence of many positive solutions of semilinear elliptic equations on annulus, J. Differential Equations 83 (1990), 348-367. MR.1033192 (91a:35073)

[3] C. V. Coffman, A nonlinear boundary value problem with many positive solutions, J. Differential Equations 54 (1984), 429-437. MR0760381 (86e:35055) 
[4] A. I. Nazarov, The one-dimensional character of an extremum point of the Friedrichs inequality in spherical and plane layers, Probl. Mat. Anal., vol. 20, Nauchn. Kniga, Novosibirsk, 2000, pp. 171-190; English transl., J. Math. Sci. (N.Y.) 102 (2000), no. 5, 4473-4486. MR1807067 (2001k:35065)

[5] N. Mizoguchi and T. Suzuki, Semilinear elliptic equations on annuli in three and higher dimensions, Houston J. Math. 22 (1996), 199-215. MR1434392 (97j:35052)

[6] J. Byeon, Existence of many nonequivalent nonradial positive solutions of semilinear elliptic equations on three-dimensional annuli, J. Differential Equations 136 (1997), 136-165. MR.1443327 (98b:35060)

[7] O. A. Ladyzhenskaya and N. N. Ural'tseva, Linear and quasilinear equations of elliptic type, 2nd ed., Nauka, Moscow, 1973; English transl. of 1st ed., Acad. Press, New York-London, 1968. MR0509265 (58:23009) MR0244627 (39:5941)

[8] R. S. Palais, The principle of symmetric criticality, Comm. Math. Phys. 69 (1979), 19-30. MR.0547524 (81c:58026)

[9] N. Trudinger, On Harnack type inequalities and their application to quasilinear elliptic equations, Comm. Pure Appl. Math. 20 (1967), 721-747. MR0226198 (37:1788)

Department of Mathematics and Mechanics, St. Petersburg State University, 28 UniverSitetskit Prospekt, Peterhoff, St. Petersburg 198504, Russia

E-mail address: sergey.kolonitskii@gmail.com

Received 22/SEP/2009

Translated by N. B. LEBEDINSKAYA 